\title{
Outcomes of grafted skin on the dorsum of the foot after car-tire friction injuries
}

\author{
Shin Hyun Kim, Won Jai Lee \\ Department of Plastic and Reconstructive Surgery, Institute for Human Tissue Restoration, Yonsei University College of Medicine, Seoul, Korea
}

\begin{abstract}
Background A car-tire friction injury on the dorsum of a child's foot often results in hypertrophic scarring of the wound margins. This study describes the clinical appearance of the injured areas and surgical complications that occurred during the follow-up period in a series of children with car-tire friction injuries who were treated with split-thickness skin grafts (STSGs). We describe the clinical features that we believe need to be highlighted when initially treating car-tire injuries in children.

Methods From May 2003 to June 2016, our retrospective study included 15 patients with car-tire injuries on the dorsum of the foot who were treated with surgical excision and STSG to cover the wound.

Results A total of 15 patients with car-tire injuries were treated. The average age was 6.26 years old. The average injury grade was 3.26. Two patients were treated using delayed repair, and 13 patients received STSG for initial management. Four patients experienced no complications, while 11 patients had hypertrophic scars and/or scar contracture after surgery.

Conclusions A car-tire friction injury on the dorsum of a child's foot often results in hypertrophic scar formation or scar contracture even if proper management is undertaken. Since the occurrence of these complications in childhood can lead to a secondary deformity, it is important to properly treat car-tire friction wounds, inform patients and caregivers about potential complications, and ensure regular follow-up evaluations over a 12-month period following the initial surgery.
\end{abstract}

Keywords Skin transplantation / Accidental injury / Hypertrophic scar

Received: May 21, $2021 \bullet$ Revised: August 3, $2021 \bullet$ Accepted: August 10, 2021

pISSN: 2234-6163 • elSSN: 2234-6171 • https://doi.org/10.5999/aps.2021.00962 • Arch Plast Surg 2021;48:678-684

\author{
Correspondence: Won Jai Lee \\ Department of Plastic and \\ Reconstructive Surgery, Institute for \\ Human Tissue Restoration, Severance \\ Hospital, Yonsei University College of \\ Medicine, 50-1 Yonsei-ro, \\ Seodaemun-gu, Seoul 03722, Korea \\ Tel: $+82-2-2228-2210$ \\ Fax: +82-2-393-6947 \\ E-mail: pswjlee@yuhs.ac
}

\section{INTRODUCTION}

Children are naturally curious, but often experience trauma due to a lack of judgment and undeveloped physical reflexes [1]. Together with the increasing number of road traffic accidents, a new type of pedestrian injury-car-tire friction injuries—has been increasingly reported in recent years. These types of injuries involve significant force, which is then transferred to the tis- sues in contact with the tire in various forms, including through friction, shear, and compression forces, causing burns and complex tissue damage on the foot [2]. As a result, the damage caused by a car-tire rolling over a body part is often considered more severe than the damage caused by more typical lacerations, abrasions, or crush injuries. Moreover, the foot has relatively tight skin, and the bony prominence at the dorsum of the foot makes proper reconstruction difficult [3]. 
Car-tire injuries are managed through similar diagnostic and treatment processes to those employed for mechanical injuries and thermal burns. However, car-tire injuries frequently involve additional friction, shearing, and compressive forces that result in more severe and deep tissue injury than may be apparent at initial presentation $[4,5]$. When a car-tire injury involves a child's lower leg, the damage due to friction may involve deep tissues in a manner that is not easily appreciated, even when the injury does not appear to be particularly severe upon the initial physical examination [1], and the formation of hypertrophic scars is a common complication [6,7]. Typically, acute complex damage to the extremities requires early debridement and coverage with healthy tissue to prevent infection, with secondary objectives of correcting functional impairment and avoiding contour deformities [8]. Many treatment options can be performed for coverage of the wound, including using a vacuumassisted closure system, skin graft, free flaps, local flaps, or crossleg flaps [9].

Hypertrophic scarring is a major complication in car-tire injuries regardless of treatment, to the point that it outnumbers infections [7]. Hypertrophic scarring has a reported incidence of $32 \%$ to $94 \%$ in patients with burn injuries and is characterized by long-lasting adverse psychological, functional, and aesthetic outcomes [10].

At our institution, we have encountered several patients with car-tire injuries who were treated with split-thickness skin grafts (STSGs), which involved a high risk of hypertrophic scar formation. This study describes the clinical appearance of the injured areas and the occurrence of surgical complications, such as hypertrophic scarring or scar contracture, during the follow-up period in a series of children with car-tire friction injuries to the foot who were treated with STSGs. Based on our experience, we also outline variables that we believe must be addressed when initially treating car-tire injuries in children.

\section{METHODS}

\section{Study design}

From May 2003 to June 2016, a total of 15 patients aged 3-10 years old with post-traumatic skin and soft tissue loss of the foot due to car-tire friction injuries were reviewed retrospectively. The patients underwent surgery between 1 and 53 days after being injured. Patient data were gathered from electronic medical records.

The following patient data were reviewed retrospectively, as shown in Table 1: age, sex, grade of injury, location and circumstances, management methods, and prognosis during follow-up. The severity of the wound was classified according to the five
Table 1. Baseline demographics and clinical characteristics of the patients analyzed in this study

\begin{tabular}{lc}
\hline \multicolumn{1}{c}{ Characteristic } & Value \\
\hline No. of patients & 15 \\
Age (yr) & $6.26(3-10)$ \\
Grade of injury ${ }^{2)}$ & $3.26(2-4)$ \\
Initial management & \\
$\quad$ Delayed repair & $2(13.3)$ \\
$\quad$ Split-thickness skin graft & $13(86.7)$ \\
Complications & \\
$\quad$ Favorable outcome & 4 \\
Hypertrophic scarring & 9 \\
$\quad$ Scar contracture & 4 \\
\hline
\end{tabular}

Values are presented as average (range) or number (\%).

a) Possible range (1-5).

grades established by Al-Qattan [1]. The injuries were assessed as follows: grade 1 injuries were superficial friction burns with no skin loss that were treatable with only a dressing, grade 2 injuries comprised deep second-degree burns accompanied by skin loss, grade 3 injuries were equivalent to third-degree burns, grade 4 injuries included exposed tendon or bone, and grade 5 injuries included severe bone loss. In addition, the aesthetic outcomes and functional recovery (range of motion and contracture) were assessed subjectively. We also examined whether patients were diagnosed with hypertrophic scars and scar contracture during long-term follow-up and whether they underwent secondary surgery (Table 2).

\section{Surgical techniques}

For the first operation, patients underwent en bloc resection until viable healthy tissue was found. Using this method, fibrous and unhealthy tissue at the margin and base of the defect was completely excised. After debridement, STSG (in some cases combined with artificial dermis) was applied to the defect. The types of commercial artificial dermis used in this study were Alloderm (LifeCell Co., Woodlands, TX, USA) and MatriDerm (Cr. Suwelack Skin \& Health Care AG, Billerbeck, Germany).

For the second operation to treat marginal hypertrophic scars, an extramarginal excision with a 3- to 5-mm margin and coverage with a full-thickness skin graft (FTSG) or STSG was performed to render mechanical tension negligible and prevent recurrence.

\section{RESULTS}

\section{Study characteristics}

A total of 15 patients were treated for car-tire injuries. Most patients suffered damage to the medial aspect of the dorsum of the foot, including three patients with ankle injuries. The average 
Table 2. Details of the patients and their treatment

\begin{tabular}{|c|c|c|c|c|c|c|c|c|c|}
\hline Sex & $\begin{array}{c}\text { Age } \\
(y r)\end{array}$ & Location of injury & Grade & First surgery & $\begin{array}{c}\text { Interval } \\
\text { between PTD } \\
\text { and first } \\
\text { surgery (day) }\end{array}$ & Complications & Second surgery & $\begin{array}{l}\text { Interval } \\
\text { between first } \\
\text { and second } \\
\text { surgery (mo) }\end{array}$ & $\begin{array}{l}\text { Follow-up } \\
\text { duration } \\
\text { (mo) }\end{array}$ \\
\hline M & 3 & Dorsum of the foot & 2 & Delayed repair & 35 & Hypertrophic scar & FTSG & 6 & 100 \\
\hline M & 7 & Dorsum of the foot $\&$ ankle & 4 & STSG with ADM & 42 & Hypertrophic scar (margin) & FTSG & 6 & 142 \\
\hline $\mathrm{F}$ & 7 & Ankle & 4 & STSG & 30 & Hypertrophic scar (margin) & FTSG & 12 & 120 \\
\hline $\mathrm{F}$ & 10 & Dorsum of the foot & 3 & STSG with ADM & 14 & Hypertrophic scar (margin) & FTSG & 14 & 50 \\
\hline M & 3 & Dorsum of the foot & 3 & STSG & 24 & $\begin{array}{l}\text { Hypertrophic scar (margin) \& } \\
\text { scar contracture }\end{array}$ & FTSG & 3 & 138 \\
\hline M & 5 & Dorsum of the foot & 3 & STSG & 1 & Hypertrophic scar(margin) & FTSG & 26 & 73 \\
\hline M & 5 & Dorsum of the foot & 3 & STSG with ADM & 27 & Scar contracture (toe) & FTSG & 40 & 90 \\
\hline M & 6 & Dorsum of the foot & 3 & Delayed repair & 10 & $\begin{array}{l}\text { Hypertrophic scar \& scar } \\
\text { contracture (toe) }\end{array}$ & $\begin{array}{l}\text { FTSG \& STSG } \\
\text { with ADM }\end{array}$ & 8 & 146 \\
\hline M & 5 & Dorsum of the foot & 3 & STSG & 30 & Hypertrophic scar (margin) & STSG with ADM & 24 & 74 \\
\hline M & 8 & Dorsum of the foot & 4 & STSG with ADM & 24 & Hypertrophic scar (margin) & STSG with ADM & 4 & 43 \\
\hline M & 3 & Dorsum of the foot \& toe & 4 & STSG with ADM & 53 & $\begin{array}{l}\text { Scar contracture (toe \& } \\
\text { dorsum of the foot) }\end{array}$ & STSG with ADM & 16 & 19 \\
\hline $\mathrm{F}$ & 10 & Dorsum of the foot & 4 & STSG with ADM & 35 & - & & & 113 \\
\hline $\mathrm{F}$ & 9 & Dorsum of the foot $\&$ ankle & 2 & STSG with ADM & 43 & - & & & 46 \\
\hline $\mathrm{F}$ & 5 & Dorsum of the foot & 4 & STSG with ADM & 24 & - & & & 22 \\
\hline M & 8 & Dorsum of the foot \& toe & 3 & STSG with ADM & 19 & - & & & \\
\hline
\end{tabular}

PTD, post-traumatic day; M, male; F, female; FTSG, full-thickness skin graft; STSG, split-thickness skin graft; ADM, acellular dermal matrix.

age of the study population was 6.26 years (range, $3-10$ years). Patients received delayed repair $(n=2)$, STSG $(n=13)$, and local flaps with STSG $(n=2)$. There were eight boys and seven girls. The average period from injury to initial treatment was 27.4 days (range, 1-53 days). Most patients underwent one or more debridement procedures during this period.

Injuries comprising several grades of severity were frequently present. The injury to a given foot was classified according to the most severe injury grade occurring within the injured zone. All wounds were determined to be grade 2 or greater. The majority of our patient population had grade 3-4 injuries, with an average grade of 3.26, and surgical treatment was performed in all cases after the wound was covered with granulation tissue.

A positive outcome was achieved in one case with grade 2 injury after debridement and an Alloderm (LifeCell Co.) skin graft on post-traumatic day (PTD) 40. However, a 3-year-old male with a grade 2 wound developed hypertrophic scarring after the first surgery.

Grade 3 injuries were identified in seven patients, and these injuries were also accompanied by fractures, dislocations, and/or tendon damage. Most grade 3 injuries were surrounded by an area with grade 2 injuries $(n=7)$. Acute damage was addressed by first removing any foreign substances from the lesion and dressing the wound until the necrotic area became clear. After treatment of bone, joint, and/or tendon damage immediately after the injury, soft tissue injuries were most frequently treated with debridement and STSG within an average of 17.85 days (range, 1-43 days). Graft acceptance was >90\%, and areas of graft loss were treated conservatively.

Grade 4 injuries were identified in six patients, all of whom had friction burns affecting the tendon and bone. Coverage was achieved using STSG after granulation tissue fully covered the defect. The median duration from injury to the first operation was 34.67 days. Despite the severe injuries in this group, complications such as infection, hematoma, and significant graft loss were not observed.

Eleven of 15 patients (73.3\%) underwent reoperation within an average of 14.45 months after the first treatment (range, 3-40 months). Hypertrophic scars were identified in nine patients and scar contracture in four patients. In two patients, two aspects, hypertrophic and scar contracture, were observed simultaneously in the wound. There were no statistically significant differences according to age, sex, injury grade, or injury date between patients who underwent reoperation and patients who did not. For both types of deformities, extralesional excision with a 3- to 5-mm margin was performed. All cases of hypertrophic scarring occurred in the margins of the initial wound, and reoperation was performed in four patients using an FTSG, and an STSG combined with acellular dermal matrix was used in four others. No specific complications were observed in eight of the patients with hypertrophic scarring after the second operation, but hypertrophic scarring recurred with scar contracture in one patient who underwent surgery a third time with an STSG. Scar contracture was most evident in the toe area, and contrac- 

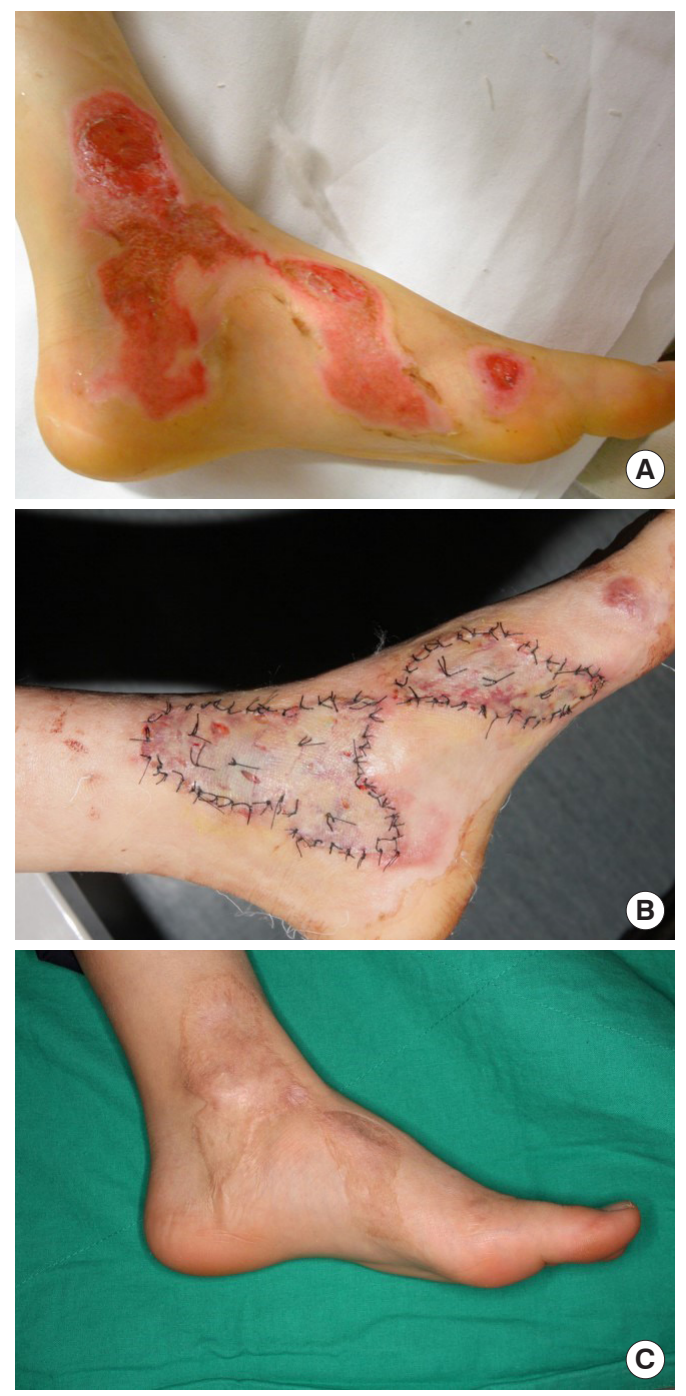

Fig. 1. An 8-year-old girl presented with a grade 2 injury. (A) The wound was clean and showed progression of granulation. (B) The granulation tissue was excised and debridement was performed. Artificial dermis with a split-thickness skin graft was used to cover the raw surface on post-traumatic day 40 . (C) No secondary deformity was evident during the 5-year follow-up period.

ture release was performed using an FTSG in most cases, with an STSG being performed in only one patient. All grafts covered the defect to render mechanical tension negligible, thereby minimizing the risk of recurrence.

The follow-up period ranged from 6 to 24 months, with an average of 12.8 months. All the patients were satisfied with the morphological appearance and functional recovery of the injured limbs.

\section{Case reports}

\section{Case 1}

An 8-year-old girl presented with a grade 2 car-tire injury (Fig. 1 ). The wound appeared to be clean, and progressive granula-
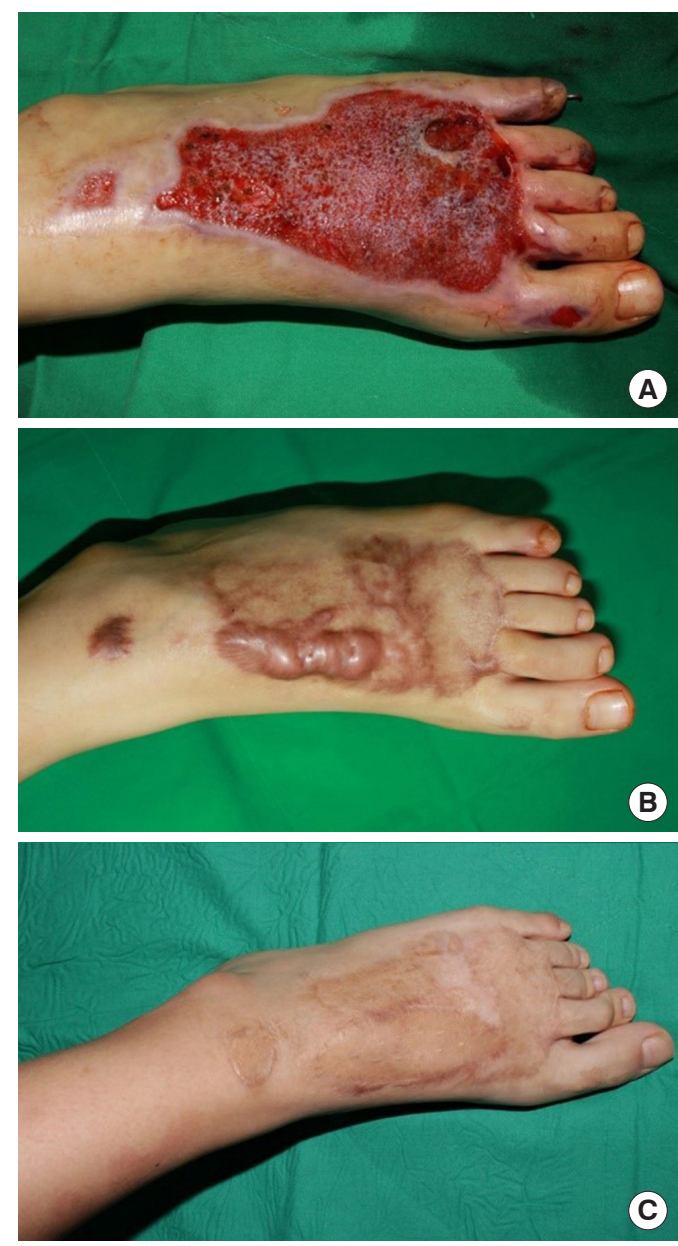

Fig. 2. A 10-year-old girl sustained a grade 2 injury on the medial side of the left ankle and foot. (A) The defect had necrotic tissue surrounded by granulation tissue. The area of the defect was excised, and artificial dermis with a split-thickness skin graft was used to cover the raw surface on post-traumatic day 15. (B) At 1 year post-surgery, hypertrophic scarring and scar contracture were evident. (C) Re-excision was performed, and the entire hypertrophic scar, together with $3 \mathrm{~mm}$ of the normal surrounding skin, was removed. No secondary deformity was evident during the 2-year follow-up period.

tion was observed with dressing changes. On PTD 40, the granulation tissue was excised and debridement was performed. Artificial dermis with an STSG was used to cover the raw surface. During the 5-year follow-up period, no secondary deformity was evident and a good surgical outcome was obtained.

\section{Case 2}

A 10-year-old girl sustained a car-tire injury on the medial side of the left ankle and foot (Fig. 2). Shortly after the injury, the defect was determined to be of grade 3 severity with a region that was mainly grade 2 . After the defect had been dressed for 2 weeks, the central area had become necrotic and was surrounded with granulation tissue. The area of the defect was excised, 
and artificial dermis with an STSG was used to cover the raw surface on PTD 15. One year after surgery, hypertrophic scarring and scar contracture were identified in the margin area in the proximal region of the dorsum of the foot. Re-excision was performed, and the entire hypertrophic scar, together with 3 $\mathrm{mm}$ of the normal surrounding skin, was removed. No secondary deformity was evident during the 2-year follow-up period.

\section{Case 3}

A 6-year-old boy presented with a grade 3 car-tire defect on the medial side of the foot, which was surrounded by a grade 2 defect (Fig. 3). On PTD 21, the patient underwent wound debridement, and the defect was covered using STSG. A hypertrophic scar and scar contracture on the dorsum of the foot were

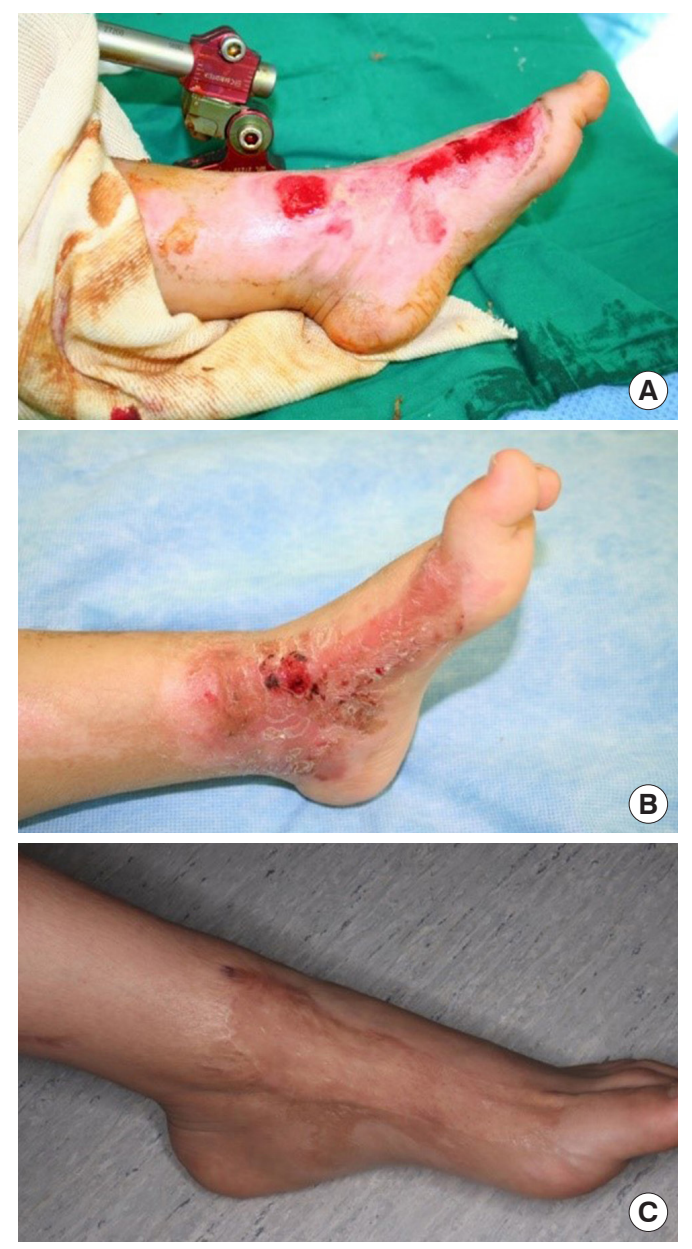

Fig. 3. A 6-year-old boy presented with a grade 3 car-tire injury on the medial side of the foot surrounded by a grade 2 defect. (A) On post-traumatic day 21, the patient underwent wound debridement, and the defect was covered with a split-thickness skin graft. (B) At 2 months after the operation, hypertrophic scarring and scar contracture on the dorsum of his foot were evident. (C) The hypertrophic scar and scar contracture band were excised and covered with a fullthickness skin graft. No secondary deformity was evident during the 10 -year follow-up period and a good cosmetic result was achieved. evident in the area of the grade 3 defect 2 months after surgery. The hypertrophic scar and scar contracture band were excised, and the defect was covered using FTSG. No secondary deformity was evident during the 10 -year follow-up period and a good cosmetic result was achieved.

\section{DISCUSSION}

The diagnosis and treatment of car-tire injuries are often similar to those of thermal burns. In deep dermal burns, the zone of coagulation, which occurs in the area closest to the heat source and is completely irreversible due to extensive protein denaturation, extends to the reticular dermis. In full-thickness burns, the zone of coagulation involves the entire dermis. In cases where there is damage to the reticular dermis, spontaneous healing is uncommon, leading to a high risk of infection due to the presence of stacked coagulated proteins and eschar formation $[11,12]$. Accordingly, early wide excision and debridement of burn wounds to remove necrotic and infected tissue and subsequent grafting are recommended to reduce the possibility of infection, promote wound healing, and reduce morbidity and mortality among patients with thermal burns $[13,14]$.

Nevertheless, damage to the foot in car-tire injuries results from both the energy of the friction burn and the energy transferred by shearing and compression [2]. In humans, who lack the panniculus carnosus that serves as a lubricant of skin and underlying tissue in animals, damage to the strongly attached skin layer may result in severe mechanical injury to deeper tissues [15]. This can be compared to thermal burn injuries, which show a gradient decrease in damage depending on the distance from the surface of the skin. Therefore, in car-tire injuries, even if the defect margin is confirmed visually after regular wound dressings and aggressive debridement, microscopic damage and injury to deeper tissue may be present. According to Shin, who evaluated the surgical margin of hypertrophic scar tissue, transforming growth factor- $\beta$-expressing fibroblasts extended along dense collagen bundles, and strongly positive proliferating cell nuclear antigen expression extending beyond the clinical margin was observed after immunohistochemical staining of excised hypertrophic scars from car-tire injuries [6]. Additionally, McDonald and Deitch [16] investigated the occurrence of hypertrophic scarring among 70 patients with thermal burn injuries who were treated with STSG and found that approximately $27 \%$ of burn sites developed hypertrophic scars after a skin graft. However, in a study of STSG use for diabetic ulcer coverage among 42 patients, hypertrophic scars developed in seven patients (16.6\%) [17]. In our study, nine out of 15 patients with car-tire injuries developed hypertrophic scars and four experi- 
enced scar contracture. This indicates that skin grafts used to cover car-tire injuries are more likely to be accompanied by complications than those used to correct defects resulting from other types of injuries, and further research is needed.

In our review of car-tire injury cases, extensive scarring in the margin of deep partial-thickness wounds was a major concern for further management, as such scarring was observed in 11 of 15 patients (73.3\%). The pathophysiology of hypertrophic scarring in burn wounds is not fully understood; however, the depth of the burn wound is known to be a crucial factor since healing takes place from the edges rather than the center of the wound, and STSGs on the hypodermis are resistant to contraction [18]. Deeper wounds may also affect adjacent structures, which may lead to more mechanical strain and additional pathologies (e.g., interactions of adipose-derived stem cells with locally produced cytokines, mechanotransduction of pain signals, and neurogenic inflammation of wound healing), which cause extensive scarring $[18,19]$. El-Gammal et al. [20] reconstructed a car-crushed dorsal muscle in the foot using a free anterolateral thigh flap in 42 children with sufficient debridement, and no marginal hypertrophic scars were found. In their study, flap bulkiness was the main complication, requiring a secondary debulking procedure, possibly due to mechanical tension and mechanotransduction near the margin.

Surgical excision of postoperative hypertrophic scars and scar contractures is generally not the preferred treatment option for adults; however, in children, soft-tissue trauma of the limbs and scar tissue formation may lead to subsequent deformities, such as an abnormal posture or gait, delayed bone growth due to the involvement of growth plates in the bones of the feet, dorsiflexion contracture, or hammer toe $[1,5,21]$. Therefore, with consideration of the contour, potential cosmetic disfigurement, and function of the foot, the injured children in our study underwent surgical interventions using STSGs after proper initial management. In this study, all 15 patients were children under the age of 10 . All of their car-tire injuries of the foot were managed according to the principles of reconstruction to remove the less viable tissue from the acutely damaged lesion and transplant healthy soft tissue for defect coverage. Eleven of the 15 patients (73.3\%) had secondary deformities and underwent reoperation after a mean interval of 14 months after the first treatment. Our results may have been influenced by the young age of the subjects, since young age is a risk factor for hypertrophic scar formation and scar contracture after skin grafting because growth causes additional tension in scarred areas [22-25]. Fortunately, there was no need for additional surgery in our patients after reoperation except for one, and all patients were satisfied with the results.

In conclusion, since car-tire injuries in children can create wide and deep tissue damage, late secondary deformities, including hypertrophic scarring and scar contracture at the repair line occurred in several patients in this study. Therefore, the extent of the damage should be accurately identified immediately after an accident, and it is important to evaluate the extent to which tissues have likely been injured; furthermore, the clinician should also consider strategies to minimize tension and postoperative pain [25]. The results of this study show that it is possible to identify and quantify the relative risk of hypertrophic skin graft development in patients with car-tire injuries. Moreover, it is important to explain potential complications such as hypertrophic scar formation and scar contracture to patients and caregivers during the preoperative stage. Regular follow-up evaluations of the wound must also be scheduled over a 1-year period after the initial skin graft, during which further interventions might be needed.

\section{NOTES}

\section{Conflict of interest}

Won Jai Lee is an editorial board member of the journal but was not involved in the peer reviewer selection, evaluation, or decision process of this article. No other potential conflicts of interest relevant to this article were reported.

\section{Ethical approval}

The study was approved by the Institutional Review Board of Severance Hospital, Yonsei University Health System (IRB No. 4-2021-1262) and performed in accordance with the principles of the Declaration of Helsinki. The informed consent was waived because this study design is a retrospective chart review.

\section{Patient consent}

The patients provided written informed consent for the publication and the use of their images.

\section{Author contribution}

Conceptualization: WJ Lee. Data curation: SH Kim, WJ Lee. Methodology: SH Kim. Writing - original draft: SH Kim. Writing - review \& editing: SH Kim, WJ Lee.

\section{ORCID}

Shin Hyun Kim https://orcid.org/0000-0003-2936-9103

Won Jai Lee https://orcid.org/0000-0003-3056-0503

\section{REFERENCES}

1. Al-Qattan MM. Car-tyre friction injuries of the foot in chil- 
dren. Burns 2000;26:399-408.

2. Nuzumlali E, Gurbuz C, Kantarci U, et al. Moving car-tire injuries of the foot: reconstruction with microvascular free flaps. J Reconstr Microsurg 1996;12:297-302.

3. Abd Al Moktader MA. Hinged multiperforator-based extended dorsalis pedis adipofascial flap for dorsal foot defects. Arch Plast Surg 2020;47:340-6.

4. Beurskens CH, van Uden CJ, Strobbe LJ, et al. The efficacy of physiotherapy upon shoulder function following axillary dissection in breast cancer, a randomized controlled study. BMC Cancer 2007; 7:166.

5. Lee SH, An SJ, Kim NR, et al. Reconstruction of postburn contracture of the forefoot using the anterolateral thigh flap. Clin Orthop Surg 2016;8:444-51.

6. Shin JU, Park J, Lee JH, et al. Extramarginal excision is preferable for hypertrophic scars. Int J Dermatol 2014;53:113844.

7. Suliman MT, Aizaz S. Car tire injuries: a review of 35 patients. Ann Saudi Med 2008;28:200-2.

8. Lee SW, Nam SK, Kim H. Friction burn on foot caused by car-tire. J Korean Burn Soc 2010;13:21-5.

9. Merter A, Armangil M, Kaya B, et al. Immediate emergency free anterolateral thigh flap after car-tyre friction injury: a case report with eight years follow-up. Int J Surg Case Rep 2017;38:102-6.

10. Finnerty CC, Jeschke MG, Branski LK, et al. Hypertrophic scarring: the greatest unmet challenge after burn injury. Lancet 2016;388:1427-36.

11. Jackson DM. The diagnosis of the depth of burning. Br J Surg 1953;40:588-96.

12. Barret JP, Herndon DN. Effects of burn wound excision on bacterial colonization and invasion. Plast Reconstr Surg 2003;111:744-50.

13. Kucan JO, Bash D. Reconstruction of the burned foot. Clin Plast Surg 1992;19:705-19.
14. Ong YS, Samuel M, Song C. Meta-analysis of early excision of burns. Burns 2006;32:145-50.

15. Billingham RE, Medawar PB. Contracture and intussusceptive growth in the healing of extensive wounds in mammalian skin. J Anat 1955;89:114-23.

16. McDonald WS, Deitch EA. Hypertrophic skin grafts in burned patients: a prospective analysis of variables. J Trauma 1987;27:147-50.

17. Puttirutvong P. Meshed skin graft versus split thickness skin graft in diabetic ulcer coverage. J Med Assoc Thai 2004;87: 66-72.

18. Wolfram D, Tzankov A, Pulzl P, et al. Hypertrophic scars and keloids: a review of their pathophysiology, risk factors, and therapeutic management. Dermatol Surg 2009;35:17181.

19. Chiang RS, Borovikova AA, King K, et al. Current concepts related to hypertrophic scarring in burn injuries. Wound Repair Regen 2016;24:466-77.

20. El-Gammal TA, El-Sayed A, Kotb MM, et al. Dorsal foot resurfacing using free anterolateral thigh (ALT) flap in children. Microsurgery 2013;33:259-64.

21. Oliveira GV, Hawkins HK, Chinkes D, et al. Hypertrophic versus non hypertrophic scars compared by immunohistochemistry and laser confocal microscopy: type I and III collagens. Int Wound J 2009;6:445-52.

22. Davies DM. Plastic and reconstructive surgery. Scars, hypertrophic scars, and keloids. Br Med J (Clin Res Ed) 1985; 290:1056-8.

23. Deitch EA, Wheelahan TM, Rose MP, et al. Hypertrophic burn scars: analysis of variables. J Trauma 1983;23:895-8.

24. Rudolph R. Contraction and the control of contraction. World J Surg 1980;4:279-87.

25. Wallace HJ, Fear MW, Crowe MM, et al. Identification of factors predicting scar outcome after burn injury in children: a prospective case-control study. Burns Trauma 2017;5:19. 